\title{
Priority medical image delivery using DTN for healthcare workers in volcanic emergency
}

\author{
Muhammad Ashar, Hirohiko Suwa, Yutaka Arakawa and Keiichi Yasumoto
}

\begin{abstract}
In this paper, targeting eye injuries caused by volcano disaster, we propose a medical image delivery service that streamlines the delivery of victim image data from a disaster area to specialist doctors in city hospitals using the Delay Tolerant Network (DTN). The service is used for an emergency response to provide quick feedback to healthcare workers after images are received by a hospital. With the received images, specialist doctors diagnose the type and seriousness of the eye injury in those images and provide appropriate medical instructions to healthcare workers. To reduce image delivery delay, it is desirable to send medical images to doctors based on image priority. For this purpose, we propose an image prioritization method in which an image is divided into pieces, and each piece is assigned a priority based on its content (for example, the severity of the injury), aiming to deliver high-priority pieces faster. Based on the priorities assigned to the pieces, we propose a message priority forwarding scheme for pieces in a DTN environment, where higher priority pieces are assigned more bandwidth and transmitted with higher resolution. Also, taking into account actual practice in a disaster area, we design and implement an application for Android devices. Through computer simulations supposing a volcano disaster scenario involving Mount Merapi in Indonesia, we confirmed that the proposed delivery service significantly shortens the image delivery time.
\end{abstract}

Keywords: Emergency response, DTN, Healthcare worker, Medical image delivery, Message priority forwarding

\section{Background}

Volcano eruptions can result in many health impacts depending on the size of the volcano. At least 500 million people worldwide live within potential exposure range to a volcano that has been active. In the case of volcanic eruption, healthcare workers will need to treat many injuries. Ash particles can affect the eyes by causing irritation or conjunctivitis as happened in the Mount St. Helen eruption and the Mount Usu eruption in Japan (Baxter et al. 2010).

In the treatment of victims, healthcare workers will sometimes need instruction from a medical specialist (an ophthalmologist) for specific eye injuries in a volcano disaster zone. In this case, communication is required between a healthcare worker inside the disaster zone and a hospital, which in many cases

\footnotetext{
* Correspondence: muhammad_ashar.Is6@is.naist.jp

Nara Institute of Science and Technology, Graduate School of Information Science, Ikoma, Nara 630-0192, Japan
}

exists outside the disaster zone, while communication equipment is impaired by such hazardous material as ash fall. In this situation, it is necessary to send medical images quickly to obtain feedback in the form of instructions that can be used by healthcare workers in the affected areas. For example, in the Mount Merapi area of Indonesia, at the time of the volcanic eruption, there is great difficulty in transmitting medical images over the large rural area to a destination in the city without mobile or wireless networks. The possibilities of travel are also very limited by the poor condition of the roads there.

The big challenge is how to deliver the most important images of injuries from a disaster zone to the city hospital faster and with good delivery performance. The opportunistic network, or Delay/Disruption Tolerant Network (DTN), is the most useful means of mobile network communication for delivering data in a disaster. In (Fujihara et al. 2014), Opportunity-based Services (OBS) provided 
evacuation guidance services using an opportunistic network. With this system, evacuees can collect smartphones location information on impassable and congested roads. In (Fajardo et al. 2014), users with mobile phones created and merged messages containing disaster-related information. This can reduce message size and minimize the overall message collection delay. In our previous study (Ashar et al. 2015), we designed a medical image delivery service over DTN with priority forwarding in a volcano disaster. This service shows better performance than existing systems in terms of message delivery rate and message delivery delay. It also delivers images of high priority faster, although the experimental scenarios in the simulation were limited.

To support medical image delivery services for healthcare workers through DTN in a volcano disaster situation, in this paper, we focus on how to make our previous work (Ashar et al. 2015) more practical by designing an efficient prioritization method of images and a data forwarding/routing mechanism, and implementing the service on prevalent mobile devices. To achieve an efficient delivery service in a volcanic emergency, first we propose a prioritization method that divides an image into high and low priority pieces. We have developed an Android application that divides each captured image into fixed-size pieces and facilitates healthcare workers in manually assigning a priority level to each piece depending on existence of the injury and its seriousness. Pieces are then sent to the destination (e.g., city hospital with a specialist) as messages via DTN. To deliver highpriority pieces (messages) faster and with better quality, we propose a priority messages forwarding scheme for DTN. In this method, first the size of each message is reduced depending on its priority (i.e., more bytes are used for a higher priority piece), and each DTN node sorts messages in its buffer in the order of their priority and sends the highest priority message to its neighboring nodes one by one through a general DTN routing protocol (e.g., epidemic routing).

Through computer simulations supposing a realistic volcano disaster, we found that the proposed method improved the message delivery rate for a fixed time interval at the hospital by up to $20 \%$ compared with the non-priority case when we use epidemic routing.

\section{Related work}

In this section, we will briefly overview existing work on applications of mobile devices for emergency situations with delivery of images through DTN.

\section{Applications for emergency situations}

Mobile devices are often used in disaster areas, especially for medical emergencies, where data are delivered by DTN. The Mobile Agent Electronic Triage Tag System (Martin-Campillo et al. 2011) creates mobile agents that store and carry triage information about victims. Mobile agents are able to move through a MANET (Mobile Ad-hoc Network) created by mobile devices without the need for an end-to-end connection from source to destination. Mobile Maps (Monares et al. 2011) presents a low-cost mobile collaborative system, which may be used in emergency situations to overcome most communication problems of firefighters. This application provides ad-hoc communication, decision support and collaboration among firefighters in the field using mobile devices. The information accumulated can be analyzed after a crisis and studied for future emergencies. The DTN implemented on Android smartphones for an emergency scenario (Wang et al. 2013) allows users to interconnect without network facilities. This study shows that a DTN node can automatically transfer to other DTN regions whatever it receives in one DTN region. It can deliver rescuers' messages including texts and videos using an epidemic routing protocol and IP Neighbor Discovery.

\section{Image-delivery services using DTN}

Photo-Net (Uddin et al. 2011) is an image-delivery service for mobile camera networks and can be used in disaster response applications. Photo-Net can send an image from the first responder who finds the victims in a disaster area by using an opportunisticforwarding scheme. CARE (Udi et al. 2012) is a system that eliminates images from a collection. It can detect the similarities among photos in DTN delivery services and optimize the capacity of the buffer on a mobile phone.

Many studies have proposed/developed applications to enhance effective communication in emergency situations. However, most of these applications do not much focus on reduction of message delivery time, which is important especially in medical image delivery services. Some existing studies such as (Joe et al. 2012); (Mashhadi et al. 2011); (Ishimaru et al. 2010) achieve timely delivery of messages by assigning priority to messages and employing a priority forwarding scheme. However, our target medical image delivery service requires good quality of medical images (at the destination), sufficient to be used for diagnosis by a specialist at the hospital. To the best of our knowledge, there are no studies on image delivery services using DTN that consider both the quality of images and reduction of delivery time. 


\section{Methods}

Medical image delivery service: target scenario and assumptions

We suppose a realistic volcanic scenario where emergency medical response teams consisting of rescuers and ambulance drivers provide services to victims. In this scenario, emergency medical response teams are treated as mobile nodes. We assume that multiple healthcare posts and one or more ambulance parking lots are located in the disaster area. At each healthcare post, a healthcare worker treats victims and takes pictures of their eye injuries using a mobile phone. Ambulance drivers move between a parking lot and a hospital to convey the victims with heavy injuries to the hospital.

In this situation, the proposed medical image delivery service aims to deliver the eye injury images of victims taken at healthcare posts to a city hospital with a specialist (i.e., ophthalmologist) and get feedback (i.e., medical instruction) for appropriate treatment of the victims.

For connections between healthcare posts and the city hospitals, the service assumes the following.

- Healthcare workers, rescuers, and ambulance drivers have mobile phones (e.g., Android smartphone) with cameras and Wi-Fi Direct communication.

- On the mobile phones, the medical image delivery application for taking and segmenting pictures of injuries and placing priority on each piece of the pictures is already installed.
The application includes mobile DTN networking software including a bundle routing protocol (e.g., (Schildt et al. 2011)) and our priority message forwarding mechanism (proposed in Sect. 4). We also assume that hospitals have network infrastructure such as an Emergency Medical Network or Wi-Fi network through which ambulance drivers can send messages stored in their phones to specialists in the hospital.

The schematic architecture of the proposed medical image delivery service is shown in Fig. 1. All images can be transferred from a disaster area to a hospital using DTN by using the proposed medical image delivery application. First, the image will be processed in the application by a healthcare worker and passed to the bundle protocol. Second, the images will be forwarded (e.g., from a rescuer's mobile phone) using Wi-Fi Direct communication to the mobile phone of another rescuer who arrives at the healthcare post and then returns to the ambulance parking area. Finally, the ambulance driver will deliver the images received from the rescuer to the city hospital, which has a communication network for sending images to the ophthalmologist at the hospital.

In the application, we also implemented a function to automatically stitch together received image patches to restore the original image so that the ophthalmologist can easily examine the image with his/her smartphone/ tablet.

\section{Priority medical image delivery scheme}

We propose a priority medical image delivery scheme with an image prioritization method used with standard

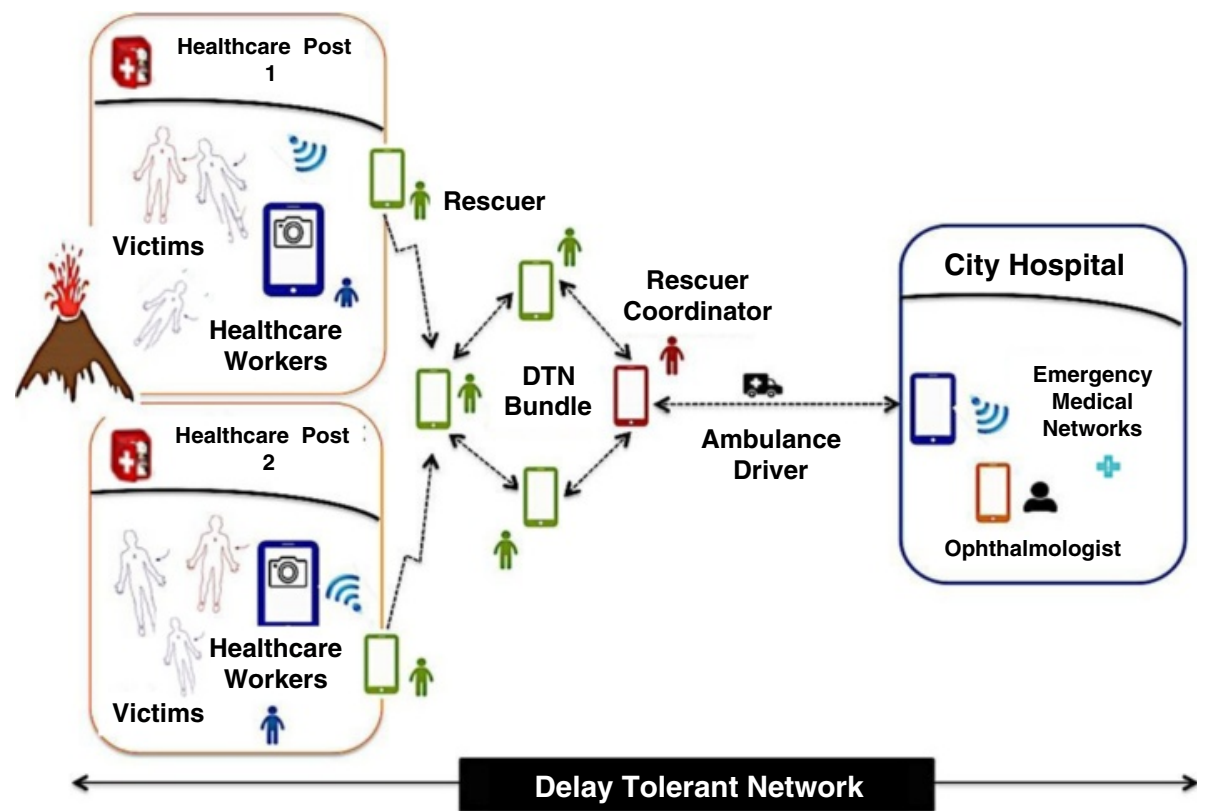

Fig. 1 Schematic architecture of medical image delivery service 
DTN routing protocols such as epidemic routing. The goal of the proposed scheme is to reduce the delivery delay and to achieve a good delivery ratio of good quality medical images delivered to a hospital per unit of time. Taking into account the mobile device's buffer size and communication capacity, we have designed strategies to build the system for efficiently delivering images in a disaster scenario.

In our proposed scheme, we assign higher priorities to more urgent images so that those images are delivered faster. Furthermore, we select the parts of images that show the most serious injuries to give them a higher priority.

\section{Assigning priorities to images}

We use a method like medical triage (i.e., color codes), for recognizing volcano victims who are in critical condition and must be brought to a hospital for immediate treatment. In disaster situations, victims are grouped into four categories, coded red, yellow, green or no color. Then, the image of each victim is partitioned into sub-blocks (Kavitha et al. 2011) by dividing up the whole image into pieces (or chunks). For efficient delivery of both high-and lowpriority pieces, we make each piece have a different data size and number of pixels depending on the color code assigned to the piece.

The color code indicates the degree of priority. Based on the seriousness of the injury, we classify the images into two groups, high-priority and lowpriority. Here, we suppose that high-priority is assigned only to the pieces including eye injury. High-priority pieces are coded with red (meaning that immediate treatment is needed), yellow (treatment can be delayed), and green (injury is minor). Low priority images have no color code.

In our method, an image is partitioned into $n \times n$ pieces. Hereafter, we suppose $n=5$, but the number can be changed. We suppose that a healthcare worker takes an eye injury image and marks some of the pieces in each image using the medical image delivery application we developed.

Figure 2 illustrates the marking process with the application where three eye injury images are taken, and, red, yellow, and green codes are given to them, respectively. The total number of high-priority pieces is 15: seven red, five yellow, and three green. The remaining 60 are of low priority. In a disaster zone without an ophthalmologist, this application can support healthcare workers by providing a way to transmit information about the symptoms of common eye diseases (De La Torre-Diez et al. 2015).

In the context of DTN application development, the message size affects the message priority forwarding strategy that will be used in our application. For a good delivery ratio in a disaster scenario, we use epidemic forwarding, which can effectively handle data up to $500 \mathrm{~KB}$ (Nikhil et al. 2015). Thus, if the message size exceeds this value, we need to reduce the size to fit this value. Therefore, in our scheme we use image quality measures and image size-reduction approaches (Kim-Han et al. 2009), (Hauswald et al. 2014) to solve this problem. Figure 3 shows an example of using the image-resizing method based on JPEG compression to reduce the size of the pieces. The application provides a blue seek-bar to adjust the quality level of the pieces of each color code between 1 and $100 \%$.

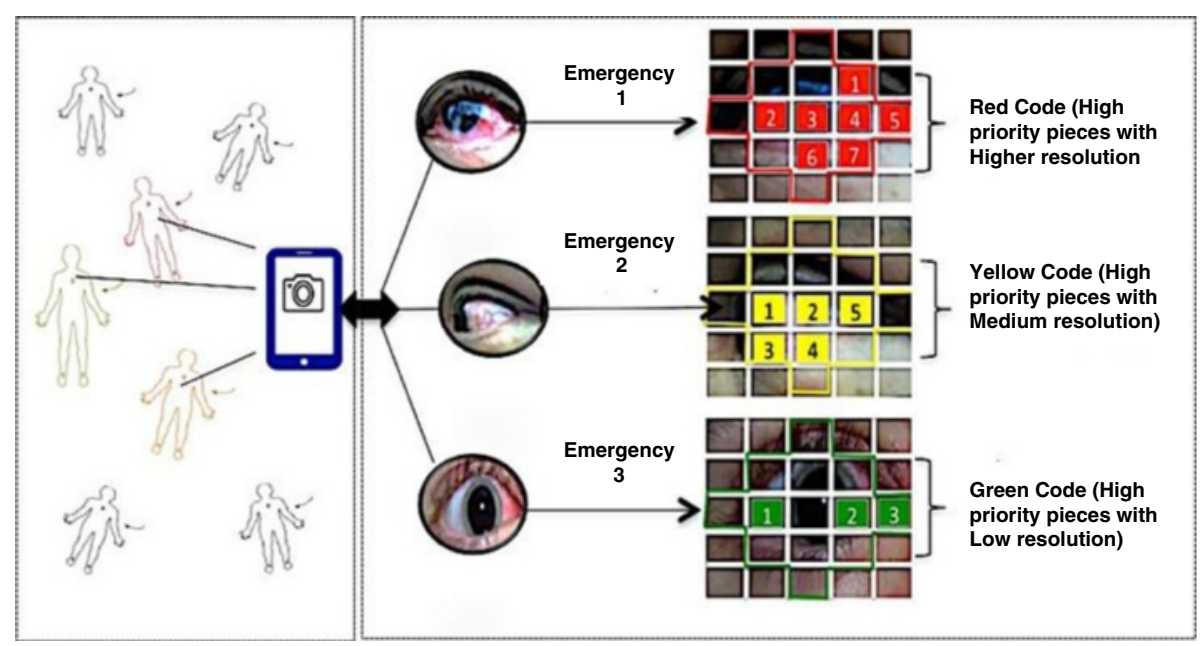

Take eye injury picture

Segmentation to decide and select high priority pieces

Fig. 2 Assigning priorities to image 


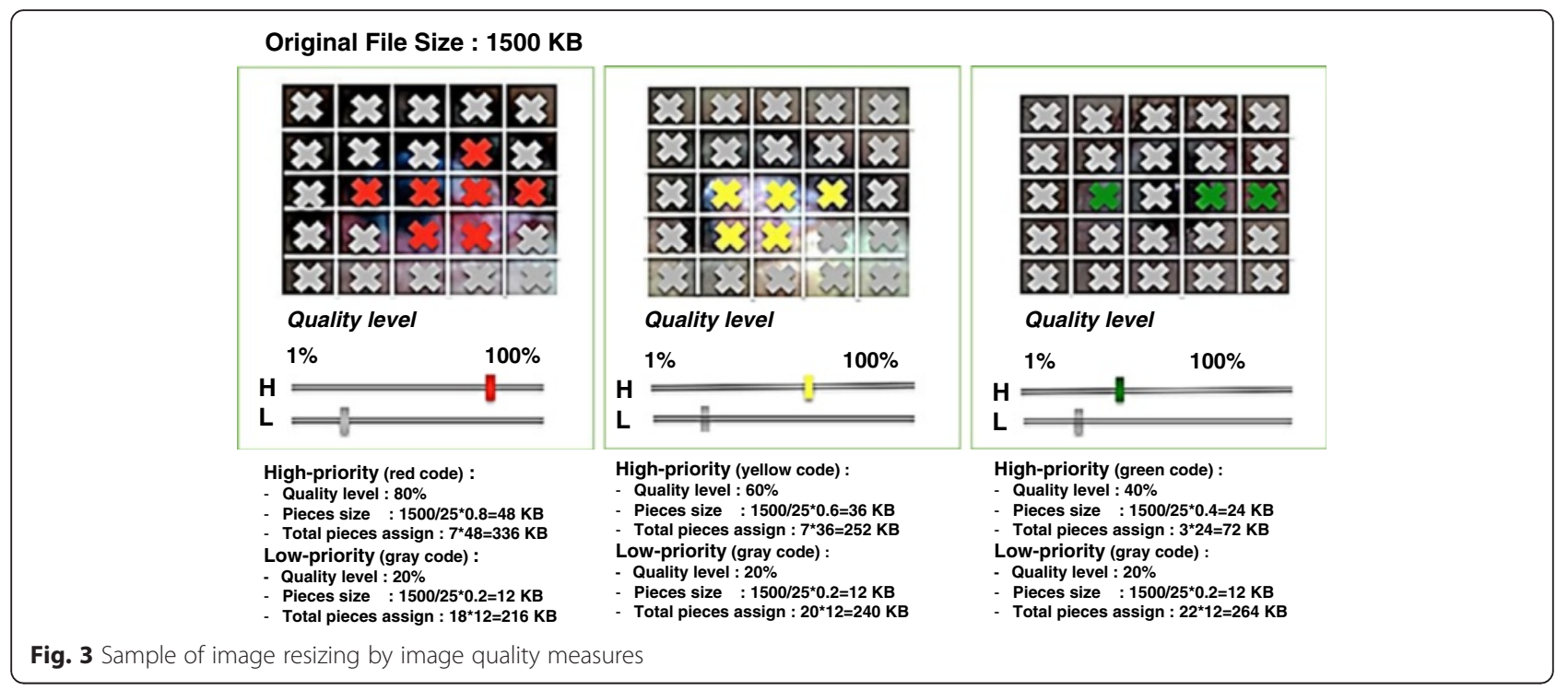

For example, if the quality level is set to $80 \%$ for red pieces, each red piece is compressed to have the file size of $80 \%$ of the original piece. That is, specifying a higher quality level maintains the good quality in pieces. In Fig. 2, $80 \%, 60 \%$, and $40 \%$ quality levels are specified for red, yellow, and green pieces, respectively. The resulting sizes of red, yellow and green pieces are $48 \mathrm{~KB}, 36 \mathrm{~KB}$ and $24 \mathrm{~KB}$, respectively.

In general, JPEG file size does not depend on its quality and we need to carefully adjust the JPEG file size. We assume that various eye injury images have similar complexity in the images, and use 1.5 MB (fixed size) for the base JPEG file size because Ref. (Robert et al. 2000) reported that ophthalmologists confirmed that this file size provides sufficient quality for diagnosis. Moreover, we use compression ratios of $80 \%, 60 \%$, and $40 \%$ for red, yellow, and green pieces of injury images, because the JPEG images compressed with these ratios still have marginal quality that can be used for diagnosis by ophthalmologists (Robert et al. 2000).

\section{Priority forwarding strategies}

The most popular routing method used in a DTN is epidemic routing. To raise the probability of messages reaching their destination, each mobile terminal copies the messages received from other terminals and holds them. This routing scheme is appropriate when the message size and generation rate are small (Takahashi et al. 2013). However, during disaster situations, images with large file size are difficult to deliver using epidemic routing. The drawbacks of epidemic routing are a rapid increase in network traffic, higher power consumption, and more terminal resource requirements. Thus, it is necessary to devise a way of reducing delivery time by prioritizing messages taking into account buffer size and the power consumption of mobile terminals. Therefore, we employ the following strategies.

1. To add a priority forwarding mechanism to epidemic routing where higher priority pieces are copied to other nodes prior to lower priority pieces.

2. Allocate a different size to each piece depending on its priority as we already explained in the previous subsection.

For strategy 1, we employ different message-handling algorithms between the healthcare worker nodes and other nodes. The details are described below.

Algorithm for healthcare worker nodes To increase message delivery rate, after each healthcare worker node generates messages (corresponding to an image), it sets TTL (e.g., 3,600 s) for those messages and sends them to the rescuer node it contacts. Each message has a chance to be sent to multiple nodes within its TTL. The messages are removed after their TTLs have expired.

Let $\mathrm{U}$ and $\mathrm{S}$ denote the set of unsent messages in the buffer, and the set of already sent messages in the buffer, respectively. Initially both $\mathrm{U}$ and $\mathrm{S}$ are empty. Whenever a new image is generated, the corresponding pieces (messages) are added to U. During the contact between the healthcare worker node and the rescuer node, messages in the buffer are handled in the following steps 1 to 3 . 
1. If $U \neq \varnothing$, then send the highest priority message $u$ $\in \mathrm{U}$ and update $\mathrm{U}$ and $\mathrm{S}$ as follows: $\mathrm{U} \leftarrow \mathrm{U} \backslash\{\mathrm{U}\}, \mathrm{S}$ $\leftarrow \mathrm{S} \cup\{\mathrm{u}\}$.

2. If $U=\varnothing$, then send the highest priority message $s$ $\in \mathrm{S}$.

3. For each $s \in S$, if TTL of $s$ has expired, then $S \leftarrow S \backslash\{S\}$.

Algorithm for other nodes Each node other than healthcare worker nodes removes messages in its buffer after sending them to another node.

- When a node meets another node, it sends the highest priority messages in the buffer during the contact.

- When a node receives messages from another node, those messages are added to the buffer and all messages in the buffer are sorted in the order of their priority (i.e., high: red, yellow, green, then low).

- When the buffer has no room for new messages, the lower priority (or the same priority but older) messages in the buffer are dropped or new messages are dropped if they have lower priority than those in the buffer.

By using these strategies, high-priority pieces are delivered to the destination faster, and at the same time, lower priority pieces can also have higher probability of being delivered than using the original epidemic routing. Below, we show how these strategies are incorporated to the proposed prioritized medical image delivery.

\section{Priority message forwarding}

As shown in Fig. 4, victims are treated at healthcare post sites (S) by healthcare workers, who need to find the shortest path for sending messages (pieces of images) to a destination (a city hospital, $\mathrm{D}_{1}, \mathrm{D}_{2}$, or $\mathrm{D}_{3}$ ). A message (m) containing a high-priority piece should be sent before other messages with lower priority. Each message is propagated by rescuers $(\mathrm{R})$ in the DTN and eventually delivered to a rescue coordinator $(C)$ stationed in the parking lot. In this case, rescue coordinators store the messages and forward them to the ambulances, and the ambulances take the volcano victims (with serious injuries) together with the messages to the hospital.

In Fig. 4, let us suppose that only one message (which includes multiple pieces) can be copied during a contact between nodes and messages $\mathrm{m}_{11}, \mathrm{~m}_{12}$, and $\mathrm{m}_{13}$ include only high priority pieces, both high and low priority pieces, and only low priority pieces, respectively. To deliver multi-priority message transmission over DTN, we employ a strategy for first selecting messages that contain pieces with a higher priority. Suppose that the source node $(\mathrm{S})$ meets multiple rescue nodes one by one. In this case, the first rescue node, which has a high memory capacity, will receive message $\mathrm{m}_{11}$ (high-priority pieces) prior to others. The second rescue node will receive a message $\mathrm{m}_{12}$ (high and low priority pieces). The third rescue node meeting the source node later will receive a message $m_{13}$ (low priority pieces). This strategy is shown in detail as follows.

Step.1 Each healthcare worker (S) node has an ordered list with a number of pieces coded with red, yellow,

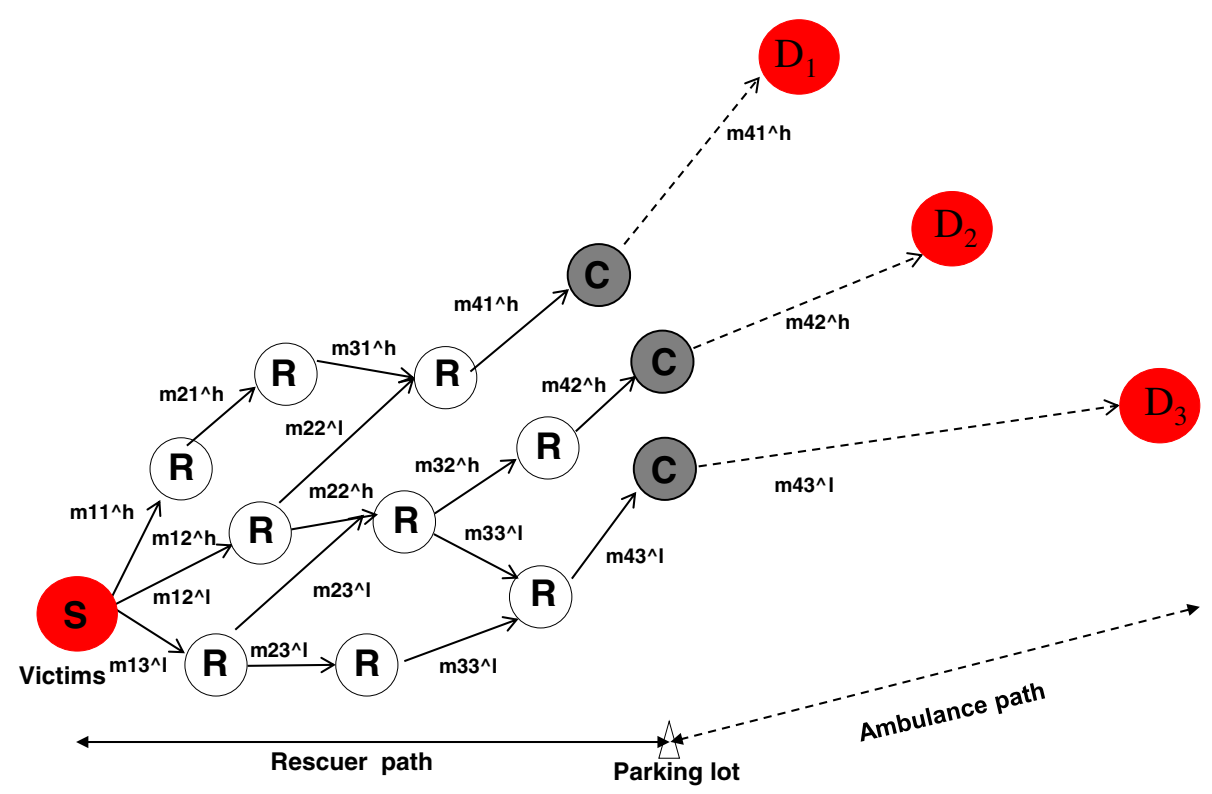

Fig. 4 Overview of DTN-based priority medical image delivery 
green, and no-color. When a contact happens, it creates a message containing multiple pieces picked from the top of the list and sends the message to the rescue (R) nodes (i.e., intermediate nodes).

Step.2 The intermediate nodes deliver the received messages to the parking lot. They keep an ordered list of the received pieces. When each intermediate node meets another node, it creates a message consisting of multiple pieces picked from the list and forwards the message during the contact.

Step.3 All messages that arrive at the parking lot are sent to the rescue coordinator $(\mathrm{C})$. The rescue coordinator (C) collects all messages, creates/updates the ordered list of the received pieces and forwards messages containing pieces picked from the list to the ambulance driver when the ambulance comes to the parking lot.

Step.4 The ambulance driver keeps the received messages until they reach a hospital (D) and sends the messages to an ophthalmologist at the hospital.

\section{Results and Discussion}

We set up a simulation experiment supposing a realistic volcanic eruption disaster map and a mobility model. Through simulations, we compare the performance of our proposed medical image delivery method with that of ordinary epidemic routing without the proposed mechanisms. To run the experiment in a realistic environment and thus simulate image delivery in an emergency situation, we use Scenargie Simulator (http:// www.spacetime-eng.com/) with the Multi-Agent Module and DTN-Dot11 Module. We present the simulation results using the volcano disaster scenario shown below.

\section{Volcano disaster scenario}

Using OpenStreetMap, we configured a simulation field on the main simulation area of $5 \mathrm{~km}$ by $5 \mathrm{~km}$ corresponding to an actual geographical area near Mount Merapi (in the region of the disaster area) and the city area (Yogjakarta, Sleman, Klaten) in Indonesia, as shown in Fig. 5. There are healthcare workers, rescuers, and rescue coordinators in the disaster area. In each city hospital in the city area, there are ambulance drivers and an ophthalmologist at each city hospital. We determined randomly the location of 20 healthcare posts where victims are treated by a healthcare worker. Each of these locations accommodates 100 people, and we assume that $5-10 \%$ of them have serious eye injuries based on the Merapi eruption situation. Rescuers walk at a normal speed between a healthcare post and a parking lot. Also, each rescuer selects a healthcare post inside the disaster area, and finds the shortest path to a parking lot. A rescuer repeatedly walks between the parking lot and the healthcare site decided at random.

We set three locations for parking lots and placed one rescue coordinator at each of these, as shown in Fig. 6.

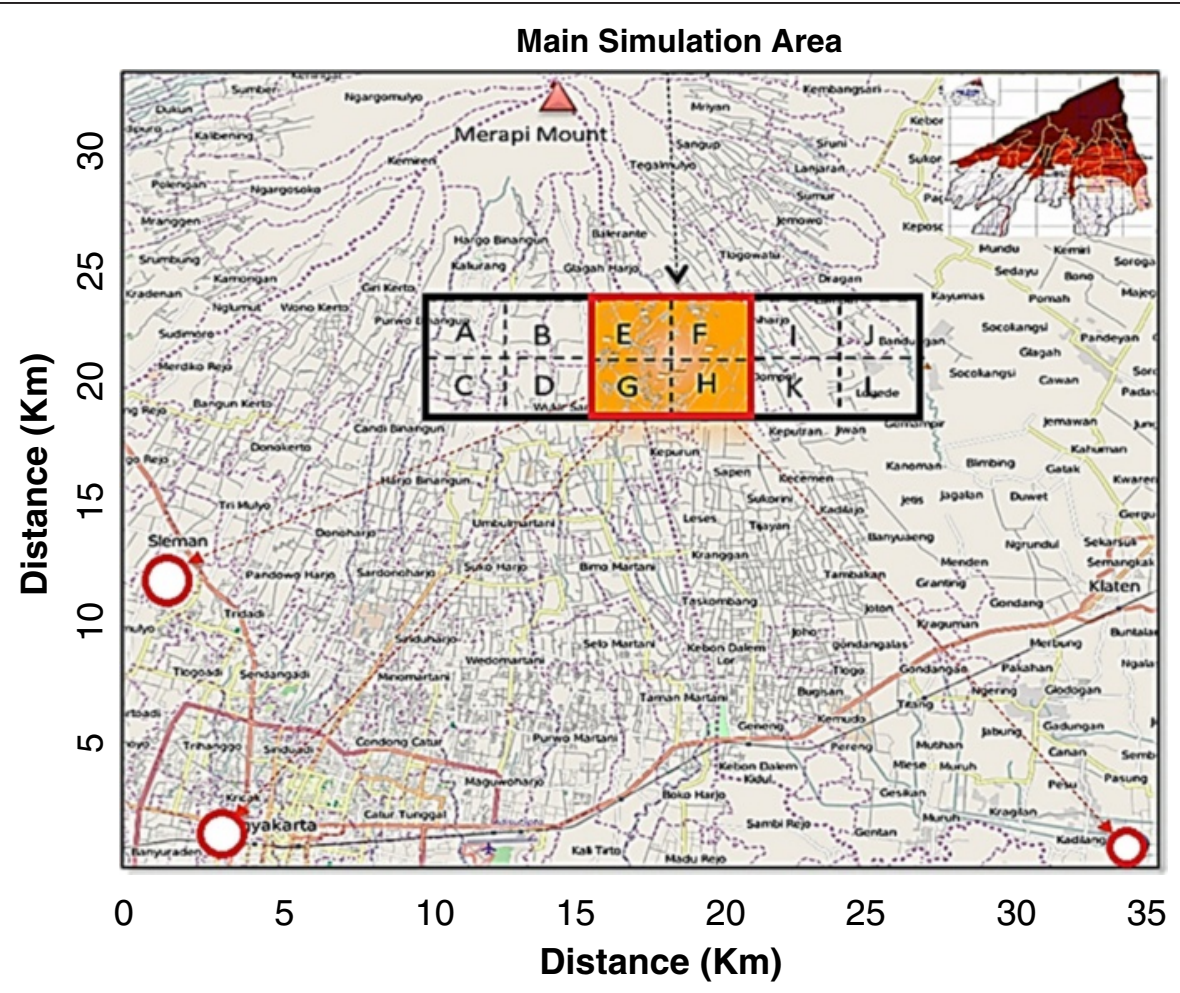

Fig. 5 Mount Merapi Volcano Situation and Simulation Area 


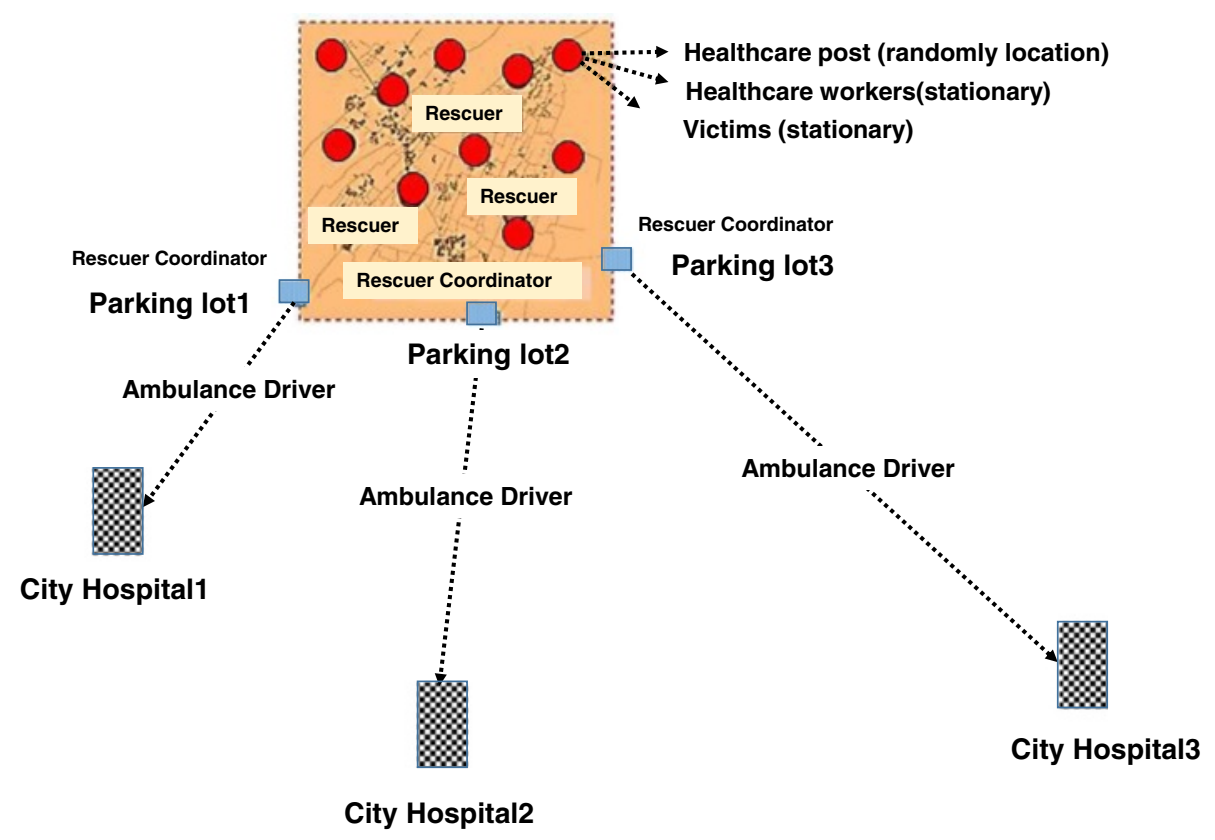

Fig. 6 Simulation field for image delivery scenario

The rescue coordinators receive the image data, do a priority sorting of the data, and store the data in an ambulance. After arriving at the parking lot and picking up the emergency victims, the ambulance driver remains there for $10 \mathrm{~min}$. During this time, the rescue coordinators transfer the image data to the ambulance driver, who then carries the messages to one of the city hospitals. The ambulance returns to the hospital from which it was originally dispatched. A contact opportunity with the ambulance comes only when the ambulance reaches the city hospital or the parking lot.

All nodes (healthcare workers, rescuers, rescuer coordinators, ambulances drivers, ophthalmologists) have mobile phones that have wireless communication capability and have installed our application. At the same time, each healthcare post has a healthcare worker who is generating images at rates of two messages per minute. The healthcare worker determines the priority of images depending on the seriousness of each injury. Each healthcare worker takes picture images with a smartphone camera and stores them in the buffer. After the images are split into pieces, they are transferred to the hospital via the parking lot.

To consider the delivery probability of data from each healthcare post to a hospital, we simulate different scenarios by changing the number of ambulances. The detailed parameters of simulation are shown in Table 1.

Testing implementation on mobile devices and applications To analyze pieces delivered between nodes through the DTN protocol, an Android mobile terminal is used to transfer the image pieces between nodes when they come in contact.

As shown in Fig. 7, we developed the DTN medical image delivery application, which can be used by healthcare workers to capture photographs and divide

Table 1 Simulation parameters

\begin{tabular}{ll}
\hline Parameter & Value \\
\hline Simulation Area & $5 \mathrm{Km} \times 5 \mathrm{Km}$ \\
Simulation Time & $2 \mathrm{~h}$ \\
Wireless Com. & IEEE $802.11 \mathrm{~g}$ \\
Transmission range & $10 \mathrm{~m}$ \\
Buffer size & $20 \mathrm{MB}$ \\
Message size: & $500 \mathrm{Byte}, 1 \mathrm{~KB}, 10 \mathrm{~KB}, 100 \mathrm{~KB}$ \\
& $1 \mathrm{MB}, 2 \mathrm{MB}$ \\
Contact times & $1 \mathrm{~s}-600 \mathrm{~s}$ \\
TTL & $3600 \mathrm{~s}$ \\
Numbers of nodes: & \\
- Victims (stationary) & 100 \\
- Healthcare workers (Stationary) & 10 \\
- Rescuer Coordinators (Stationary) & 3 \\
- Ophthalmologists (Stationary) & 3 \\
- Rescuers & $20,40,60,80,100,120$ \\
- Ambulances Drivers & $3,6,9,12,15$ \\
Mobility speeds: & \\
- Pedestrian (rescuers) & $1-2 \mathrm{~m} / \mathrm{s}$ \\
- Vehicle (ambulances) & $5-12 \mathrm{~m} / \mathrm{s}$ \\
- Distance to hospital & $20 \mathrm{Km}, 30 \mathrm{Km}, 40 \mathrm{~km}$ \\
\hline
\end{tabular}




\section{Image processing part}
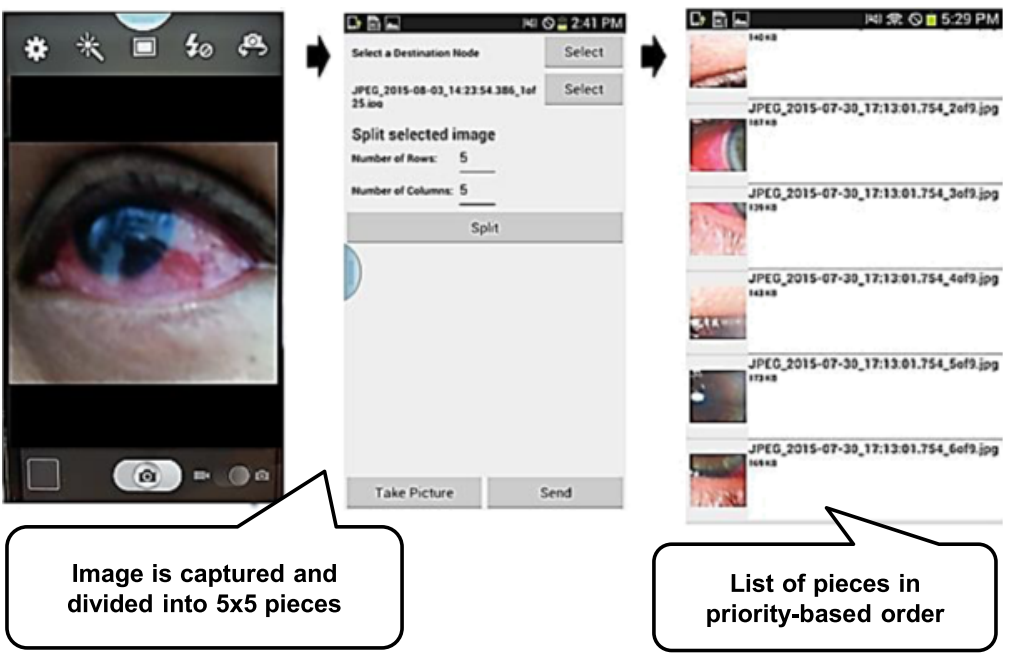

DTN sending part
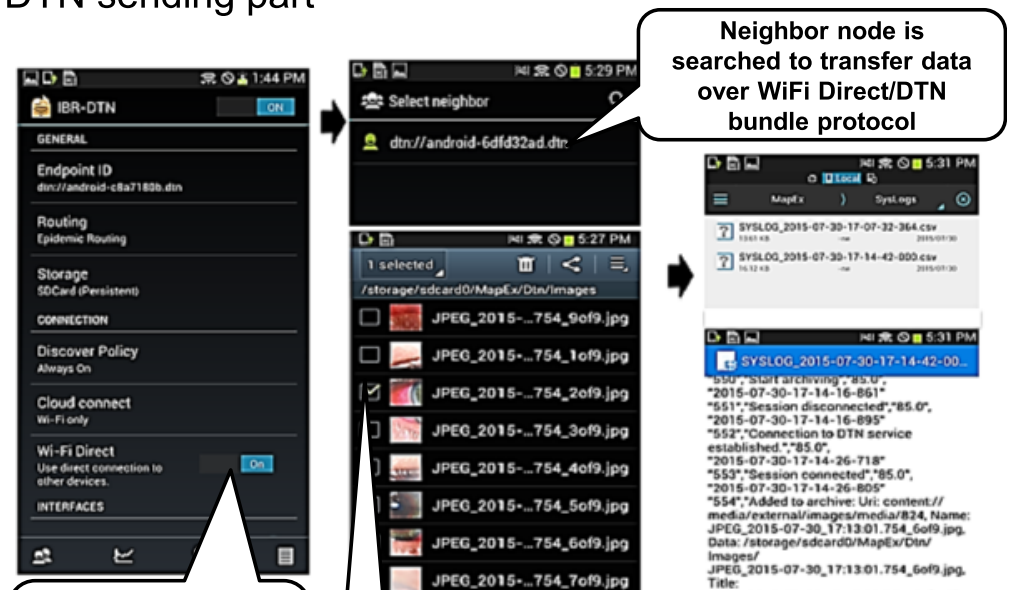

DTN configuration (routing protocol and communication type)
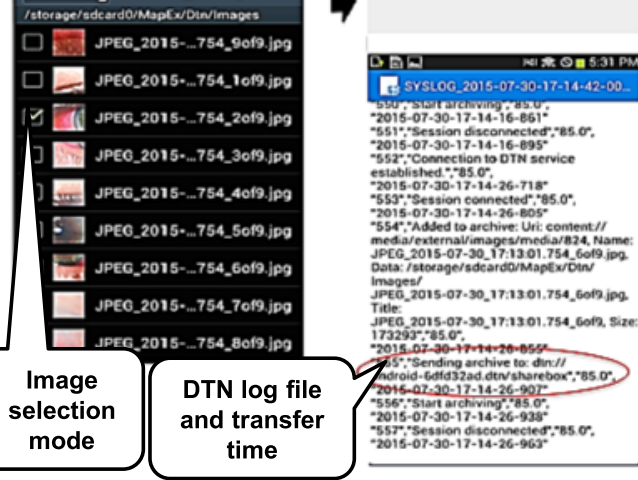

DTN receiving part

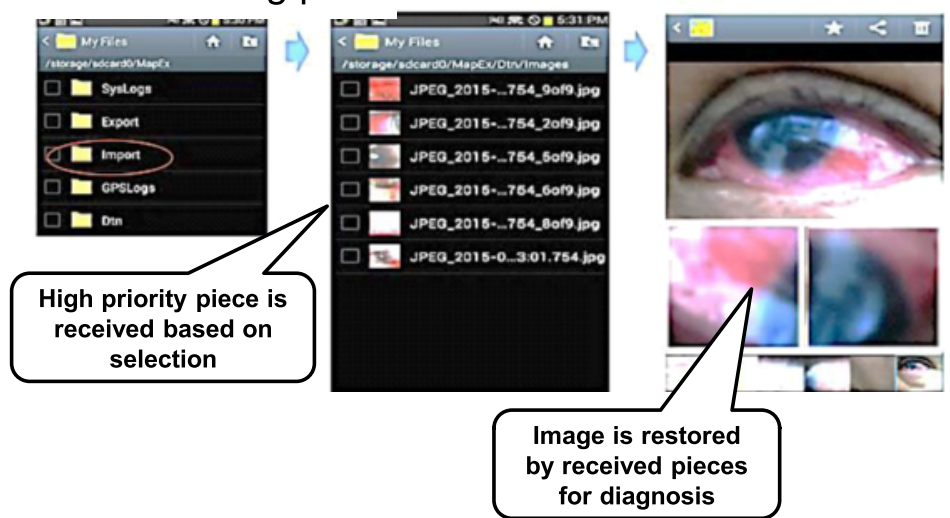

Fig. 7 User interface of medical image transfer by mobile application 
images into pieces. Pieces are stored in high-and lowpriority files to be easily selected and forwarded as prioritized images using an IBR-DTN Android implementation (Morgenroth et al. 2012).

With the user interface of the mobile application, users (healthcare workers) manually capture images of an eye of a victim. A user can easily recognize the seriousness of an injury and determine the priority of the image pieces by using the interface. Then, all pieces are stored and forwarded using a DTN bundle protocol. Finally, the pieces are sent based on their priorities to facilitate the diagnosis using the image by an ophthalmologist. The pieces received at the hospital can then be merged.

We assume that the ophthalmologist in the hospital has a smartphone in which our app is already installed. Therefore, when all (or part of) image patches arrive at the destination (final user, that is, the ophthalmologist), they will be automatically stitched together to restore the original image.

Here, note that the missing (not-received) pieces in the restored image remain blank.

\section{Results}

In this section, we evaluate the message delivery rate with respect to time, message size and number of mobility nodes (ambulance drivers, rescuers).

Figure 8 shows how the delivery rate of a message is varied over an interval of two hours when the number of rescuers is 60 and the message size is 500 Bytes. Each simulation is conducted four times and averaged.
Moreover, to know the impact of each parameter, we changed the message size from $1 \mathrm{~KB}$ to $2 \mathrm{MB}$, the number of mobility nodes from 20 to 100 rescuers and the number of ambulances from 3 to 15, which deliver messages from the disaster area to the city hospital on three-lane roads. We show the results in Figs. 9 and 10, respectively. In all cases, the message delivery rate when priority is considered is higher. In Fig. 10b, it is clearly seen that the message delivery rate is quite high when giving priority to the image of eye injury as well as increasing the number of rescuers and ambulances.

\section{Discussion}

We performed a medical image transmission analysis on a simple mobility scenario in a volcano disaster area to determine the successful delivery ratio using a mobile wireless link. The available time that a node can use for data transfer is based on priority and the message forwarding strategies in an environment where only DTN or opportunistic links are available. The performance metrics we considered are of two types: (a) the first one is measuring a good probability of delivering a message taking into account additional variation of parameter settings such as message size, number of intermediate nodes, and ambulances nodes; (b) the second type is to measure the actual time of transferring a medical image with the implementation on the mobile devices using a prioritized image and DTN protocol.

Figure 8 shows the message delivery rate. There are four points at which the delivery rate increases. The high-priority pieces' delivery rate is approximately $41 \%$

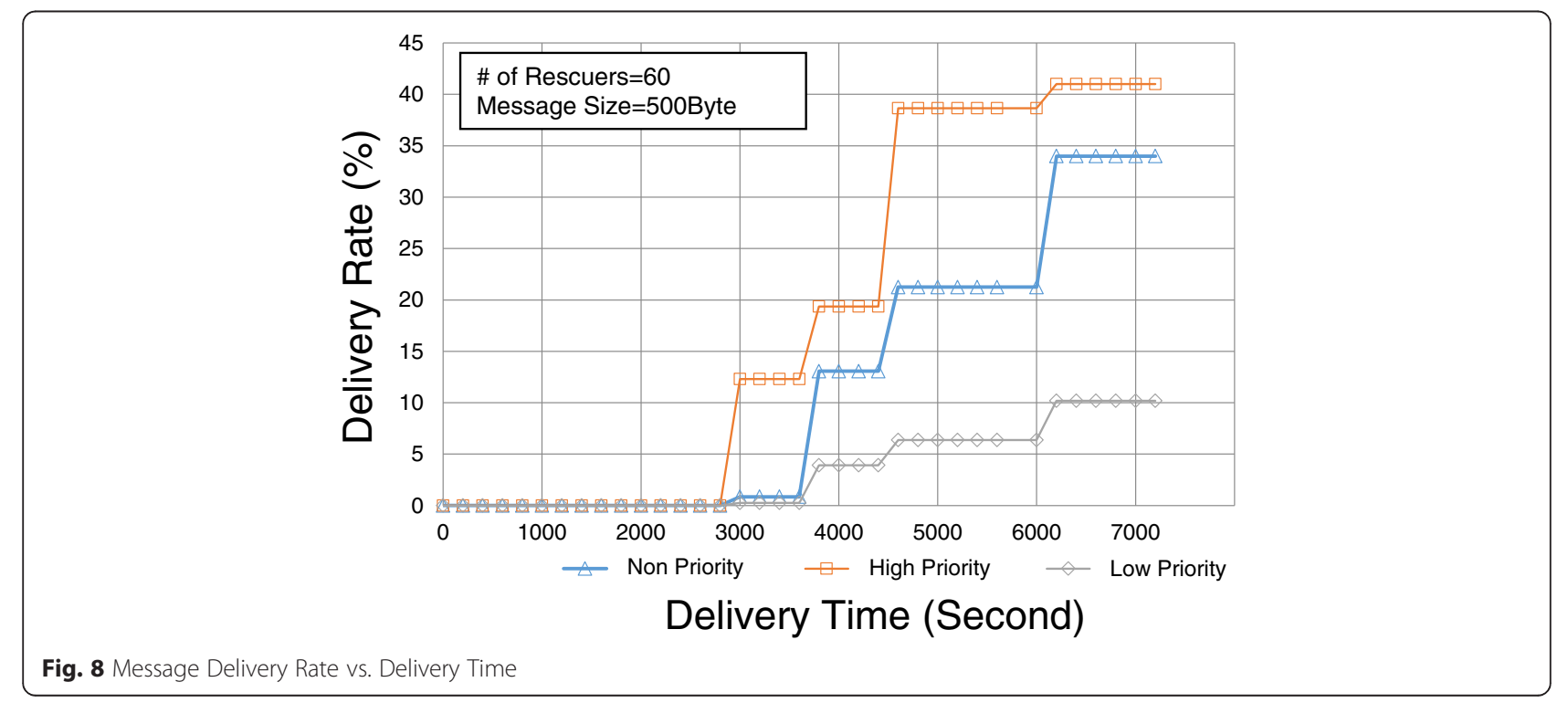



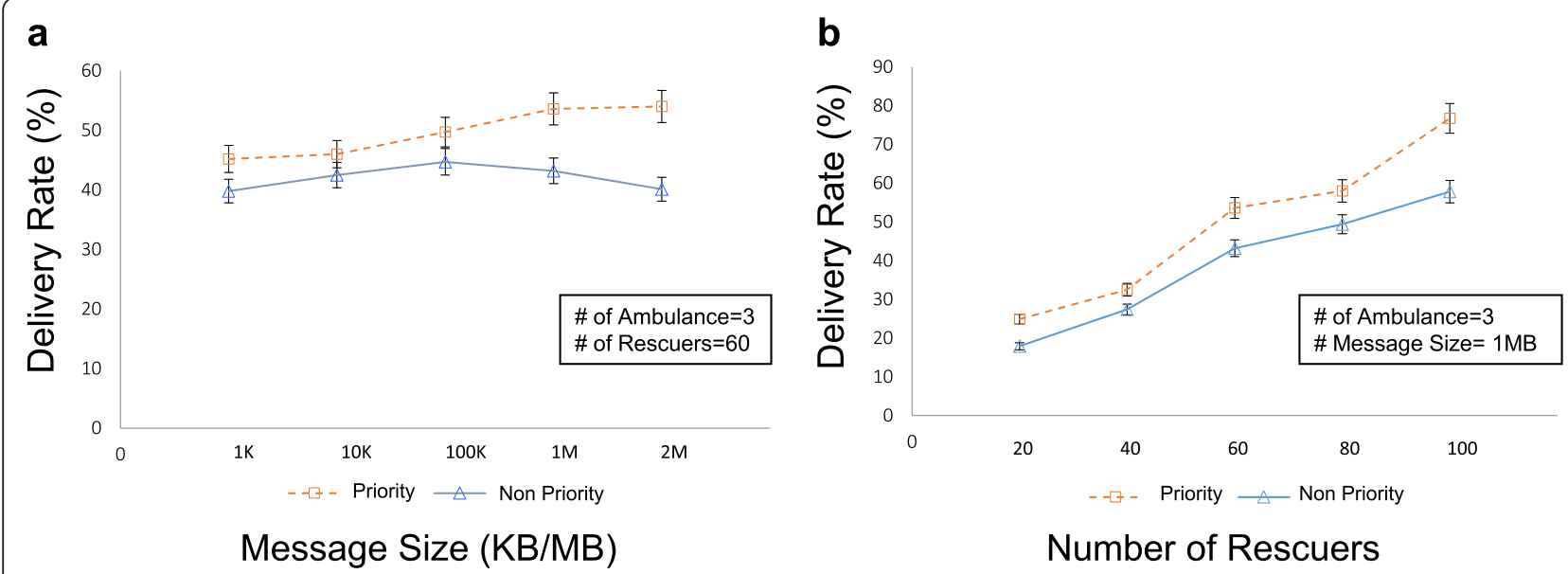

Fig. 9 Message Delivery Rate vs. Message Size, Number of rescuers

at the end of the simulation. In other cases, non-priority pieces' delivery rate is $34 \%$, whereas the low priority pieces' rate is about $10 \%$. As a result, the proposed method can deliver a message faster than the conventional method (i.e., epidemic routing without a priority mechanism). In this case, each ambulance has a different route and distance to travel from the hospital to the parking lot in the disaster area. In the figures, the performance of the proposed method is shown for highpriority and low-priority delivery, while non-priority delivery corresponds to the conventional method (epidemic routing without prioritization).

By evaluating the results in Fig. 9a, it can be observed that there is a significant improvement (10\%) in the message delivery rate using prioritization if there is an increase in message size. These results are due to a better choice of high-priority messages. That is, based on the proposed forwarding scheme, the message with the highest priority should be on top of the queue and be forwarded before the others. The messages with high-priority images, tend to reach their destination faster, thus keeping the average delay lower.

According to the results (Figs. 9b and 10a), it can be observed that both priority and non-priority images can have better delivery rates as the number of mobile nodes such as rescuers or ambulance drivers increases. This is because the contact opportunity between mobile nodes increases as the number of the mobile nodes increases.

Many messages with a low priority could not be delivered due to the limited capacity of the ambulance when the number of ambulances is small. Therefore, we increased the number of ambulances and the number of rescuers. As a result, the message delivery rate increased from $76 \%$ to $90 \%$ by the end of the simulation (Fig. 10b). This shows that $90 \%$ of all 60 generated images could be delivered as a result of the increased frequency of ambulances coming to the parking lot.

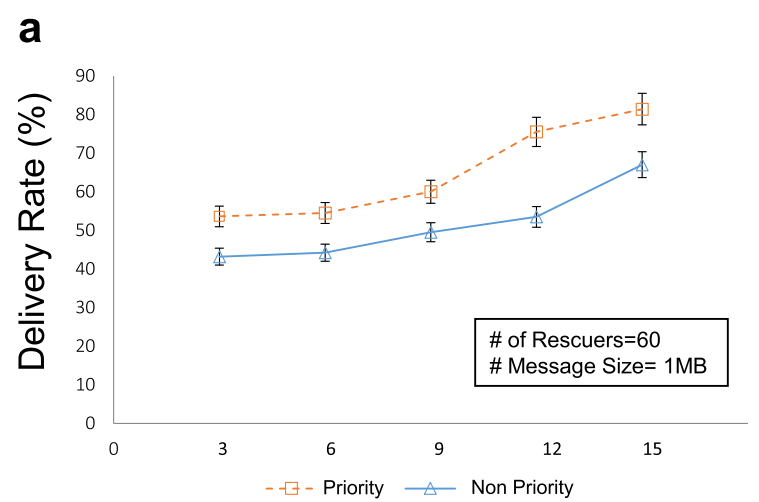

Number of Ambulances

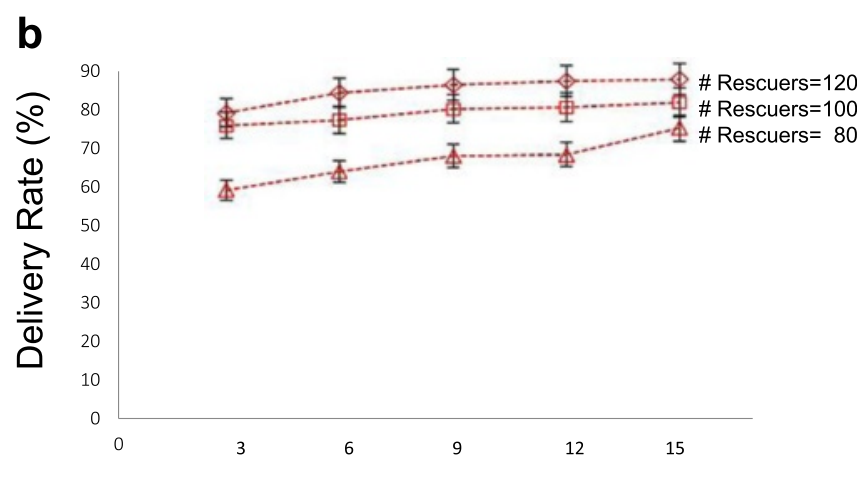

Number of Ambulances

Fig. 10 Message Delivery Rate vs. number of Mobility Nodes (ambulances) 
Table 2 Time transfer measurement for priority images

\begin{tabular}{|c|c|c|c|c|}
\hline \multirow[t]{2}{*}{ Users } & \multirow[t]{2}{*}{ Image size in KB } & \multicolumn{2}{|c|}{ \# of pieces } & \multirow[t]{2}{*}{ Time in seconds } \\
\hline & & High & Low & \\
\hline \multicolumn{5}{|l|}{ A } \\
\hline Image1 & 1890 & 7 & 6 & 74 \\
\hline Image2 & 1640 & 5 & 8 & 61 \\
\hline Image3 & 1310 & 3 & 10 & 50 \\
\hline \multicolumn{5}{|l|}{ B } \\
\hline Image4 & 1920 & 8 & 5 & 85 \\
\hline Image5 & 1685 & 5 & 8 & 68 \\
\hline Image6 & 1380 & 4 & 9 & 57 \\
\hline \multicolumn{5}{|l|}{ C } \\
\hline Image7 & 1845 & 7 & 6 & 72 \\
\hline Image8 & 1610 & 5 & 8 & 67 \\
\hline Image9 & 1275 & 4 & 9 & 51 \\
\hline \multicolumn{5}{|l|}{ D } \\
\hline Image10 & 1935 & 8 & 5 & 82 \\
\hline Image11 & 1630 & 6 & 7 & 70 \\
\hline Image12 & 1395 & 4 & 9 & 60 \\
\hline \multicolumn{5}{|l|}{$E$} \\
\hline Image13 & 1870 & 7 & 6 & 75 \\
\hline Image14 & 1625 & 5 & 8 & 66 \\
\hline Image15 & 1370 & 3 & 10 & 51 \\
\hline
\end{tabular}

To evaluate the actual transferring time on the medical delivery service implementation, we have measured an average time transfer for each piece in a priority image between two Android devices. We asked five participants to join the experiment. Each participant was asked to transfer three images with injuries of varying seriousness and of different sizes. The total number of pieces to be transmitted (of high and low priority) was 13 pieces. As shown in Table 2, the average transfer time for the 1st, 2nd and 3rd images were $77.6 \mathrm{~s}, 66.4 \mathrm{~s}$ and 53.8 s,respectively. The transfer time decreases as the users get used to do the transfer process. The transfer time was greater when the number of high-priority pieces exceeded the number of low priority pieces.
A showcase timeline of the total time transfer measurement process is presented in Fig. 11. The generic steps were: (1) record the image processing time (T1); (2) send pieces copy file to send DTN bundle delivery time (T2); (3) start image transfer to other device time (T3); (4) store pieces copy file to receive DTN bundle delivery time (T4) and view images kept in storage (T5). The areas marked in a gray block represent the time slots relevant for our measurement. The average times of total image transfer are shown in Table 2.

\section{Conclusion}

Based on priorities in medical image delivery through DTN in a volcano disaster scenario, we noted that the message delivery time could be shortened by using message priority routing strategies and prioritizing images according to the seriousness of injuries. We have improved the delivery rate in the simulated experiments by increasing the number of mobility nodes (rescuers and ambulances drivers).

In this paper, we described the performance of medical image delivery using existing DTN protocols in a disaster situation. We proposed a message priority forwarding scheme, which is a modification of an epidemic routing protocol. The proposed method is designed to increase the delivery rate of high-priority messages so as to increase the number of messages delivered by healthcare workers at a volcano disaster. The results were evaluated using simulations and by implementation on mobile devices. The outcome showed faster delivery of medical images in the emergency scenario, and the number of successfully delivered images with prioritization that could be used for diagnosis at a hospital was higher than with a conventional method (epidemic routing without prioritization).

As part of future work, we will improve the proposed service in terms of efficiency of selecting high-priority pieces. For example, automating high-priority pieces selection through image analysis may be worth exploring; it could reduce the burden on healthcare workers at the disaster sites to a great extent.

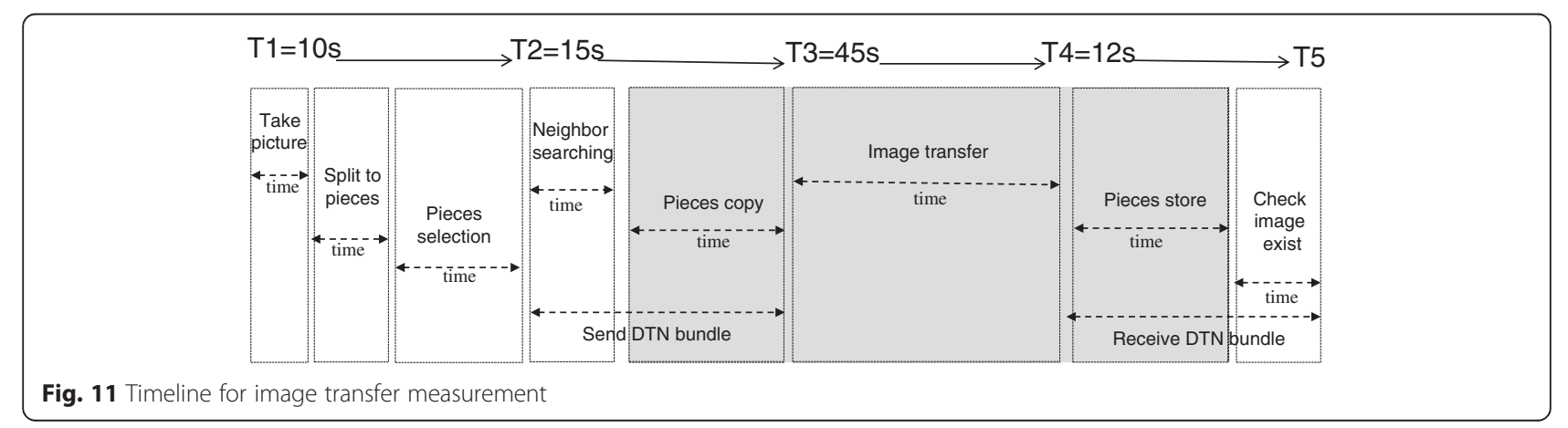




\section{Competing interest}

The authors declare that they have no competing interests.

\section{Authors' contribution}

The authors have contributed to this paper and all authors read and approved the final manuscript.

\section{Acknowledgements}

Part of this work has been supported by JSPS Kakenhi Grant Number 2622001 and DIKTI scholarship funding from Indonesia Government.

Received: 18 January 2016 Accepted: 3 May 2016

Published online: 19 July 2016

\section{References}

Ashar M, Tamai M, Arakawa Y, Yasumoto K. Prioritized Medical Image Forwarding over DTN in a Volcano Disaster, International Conferences Advanced Multimedia \& Ubiquitous Engineering, Future Information Technology (MUE), Lecture Note Electrical Engineering, Vol. 354, p. 431-441. Springer, (2015)

Baxter PJ, Bernstein RS, Falk H, French J, Ing R. Medical Aspects of Volcanic Disaster: An outline of Hazards and Emergency Response Measures. In: Disasters, Vol. 6, No. 2, p. 268-276, Wiley-online library (2010)

De La Torre-Diez I, Martinez-Perez B, Lopez-Coronado M, Diaz JR, Lopez MM. Decision Support Systems and Application in Ophthalmology: Literature and Commercial Review Focused on Mobile Apps. J Medical Systems. 2015; 39(174): $1-10$

Fajardo J, Yasumoto K, Shibata N, Sun W, Ito M. Disaster Information Collection with Opportunistic Communication and Message Aggregation. Journal of Information Processing. 2014;22(2):106-17.

Fujihara A, Miwa H. Disaster Evacuation Guidance using Opportunistic Communication: The Potential for Opportunity-Based Services. In: Big Data and Internet of Things: A Roadmap for Smart Environments Studies in Computational Intelligence, Vol. 546, p. 425-446, Springer, Switzerland (2014)

Hauswald J, Manville T, Zheng Q, Dreslinski R, Chakrabarti C, Mudge T. A Hybrid Approach To Offloading Mobile Image Classification. In: IEEE Int'I Conf. on Acoustic, Speech and Signal Processing (ICASSP), p. 8375-8379 (2014)

Ishimaru Y, Sun W, Yasumoto K, Ito M. DTN-based Delivery of Word-of-Mouth Information with Priority and Deadline. In: Proc. of the $5^{\text {th }}$ International Conference on Mobile Computing and Ubiquitous Networking (ICMU), p. 179-185 (2010)

Joe I, Sang-Bo K. A Message Priority Routing Protocol for Delay Tolerant Networks (DTN) in Disaster Areas. In: Future Generation Information Technology, LNCS, Vol. 6485, p. 727-737, Springer, Berlin (2012)

Kavitha C, Rao BP, Govhardan A. Image Retrieval Based on Color and Texture Features of the Image Sub-blocks. In: Int'l J. of Computer Application, Vol. 15, No. 7 (2011)

Kim-Han T, Raveendran P. A survey of image quality measures. In: IEEE Int'I Conf. Technical Postgraduates (Techpos), p. 1-4, Kualalumpur (2009)

Martin-Campillo A, Marti R, Yoneki E, Crowcroft J. Electronic Triage Tag and Opportunistic Network in Disaster. In: Proc. of special workshop on Internet and Disasters, No. 6, Tokyo (2011)

Mashhadi AJ, Capra L. Priority Scheduling for Participatory Delay Tolerant Networks. In Proc. of the $12^{\text {th }}$ IEEE International Symposium on a World of Wireless, Mobile and Multimedia Networks (WoWMoM), p. 1-3 (2011)

Monares A, Ochoa SF, Pino JA, Herskovic V, Rodriguez-Covili J, Neyem A. Mobile Computing in Urban Emergency Situation: Improving the Support to Firefighters in the Field. J Expert System with Applications. 2011;38:1255-67. Elsevier.

Morgenroth J, Schildt S, Wolf L. A Bundle Protocol Implementation for Android Devices. In: Proc. ACM the $18^{\text {th }}$ annual Int'l. Conf. on Mobile Computing and Networking (Mobicom), p. 443-446 (2012)

Nikhil G, Shital NG. Evaluation of DTN Routing Protocols in Post Disaster Scenario. In: Int'l J. of Computer Application, Vol. 121, No. 18 (2015)

Robert HE, Kanagasingam Y, Chris JB, lan JC, Mei-ling TK, Ludmila J, Philip HH. Methods and Limits of Digital Image Compression of Retinal Image for Telemedicine, Investigative Ophthalmologist \& Visual Science, vol., 41, No. 7, p. 1916-1924, (2000)

Schildt S, Morgenroth J, Pottner WB, Wolf L. IBR-DTN: A lightweight, modular and highly portable Bundle Protocol implementation. Electronic Communications of the EASST, Vol. 37, p. 1-10 (2011).
Takahashi A, Nishiyama H, Kato N. Fairness Issue in Message Delivery in Delay and Disruption Tolerant Network for Disaster Areas. In: Proc. Int'I Conf. on Computing, Network, and Communications (ICNC), p. 890-894, USA (2013) Uddin MYS, Wang H, Saremi F, Qi G, Abdelzaher T, Huang T. PhotoNet: A Similarity-aware Picture Delivery Service for Situation Awareness. In: Proc. Of IEEE Real Time Systems Symposium (RTSS). p. 317-326 (2011)

Udi W, Qingxi L, Nina T, Athula B, Vyas S, Gianluca I, Srinivasan S. CARE: Content Aware Redundancy Elimination for Disaster Communications on Damaged Networks. In: Proc. Of the $11^{\text {th }}$ ACM Workshop on Hot Topics in Networks (HotNets), p. 127-132 (2012)

Wang Y, Yu C. Implementing Delay Tolerant Networks on Android Devices in Emergency Scenario. Sciencepaper Online, p. 1-10 (2013). http://www.paper. edu.cn/index.php/default/en_releasepaper/downPaper/201303-665. Accessed 20 May 2016.

\section{Submit your manuscript to a SpringerOpen ${ }^{\circ}$ journal and benefit from:}

- Convenient online submission

- Rigorous peer review

- Immediate publication on acceptance

- Open access: articles freely available online

- High visibility within the field

- Retaining the copyright to your article

Submit your next manuscript at $\boldsymbol{s p r i n g e r o p e n . c o m ~}$ 